\title{
AN EFFICIENT METHOD FOR YIELD AND FAILURE SURFACES OF THE STEEL I- SECTION
}

\author{
Anh $\mathrm{Q} V \mathrm{Vu}^{1,{ }^{*}, \text { Nghia H Hoang }}{ }^{2}$, and Hien M Nghiem ${ }^{1}$ \\ ${ }^{I}$ Department of Civil Engineering, Hanoi Architectural University, Hanoi Vietnam \\ ${ }^{2}$ Department of Civil Engineering, Haiphong University, Haiphong Vietnam \\ *(Corresponding author: E-mail: anhvq@hau.edu.vn)
}

\section{A B S T RA C T}

The paper presents a new method to generate efficiently yield and failure surfaces of the doubly symmetrical wide flange I-section under axial force combined with biaxial bending moments in nonlinear analysis of the steel frame. The I-section is divided into several rectangular areas, and the axial force is integrated from uniform stress on each area to form the equilibrium equations of the failure surface. Hyperbolic equations are proposed to approximate the biaxial bending moments of gradually yielding cross-section that represent the yield surfaces lie between limit elastic and failure surfaces. A computer code SPH developed with the implementation of the proposed method aims to perform analyses for numerous cross-sections. The proposed method may help to improve the effectiveness of the finite element analysis of the steel frame in comparison to the fiber method. Several computational examples are conducted to validate the accuracy and efficiency of the proposed method by comparing the results predicted by SPH with those retrieved from other methods.
A R T I C LE H I S T O R Y

$\begin{array}{ll}\text { Received: } & \text { 4 October } 2019 \\ \text { Revised: } & \text { 30 April } 2020 \\ \text { Accepted: } & \text { 30 May } 2020\end{array}$

\section{K E Y W O R D S}

yield surface;

failure surface;

biaxial bending;

steel I-section;

\section{Introduction}

The yield and failure surface concepts had been widely used in inelastic frame analysis to model the full plastification of the steel section under axial force combined with biaxial bending moment (Duan and Chen [1]). Deriving closed-form equations theoretically for the yield and failure surfaces of a general section is complex. For a practical structural analysis, the yield and failure surfaces should be approximated by several simple equations. In the literature, several methods have been presented for the ultimate strength analyses of various sections, such as wide-flange I-section (Orbison et al. [2]), rectangular, circular, and I-sections (Duan and Chen [1]) under axial force combined with biaxial moments. Orbison et al. [2] proposed a polynomial expression for failure surface that contained ratios of axial force and bending moments to the corresponding squash load and plastic moments. Duan and Chen [1] developed smooth and convex failure surfaces in the three-dimension space that satisfied all special cases.

The most promising model for nonlinear analysis of the beam-column element is the fiber method (Taucer et al. [3]). In this method, the cross-section is usually discretized into small fibers, and the nonlinear behavior of the material is represented by the uniaxial stress-strain relationship of a fiber (NgoHuu [4], Ngo-Huu and Kim [5], and Nguyen and Kim [6]). Since the fiber method is the most computationally intensive but more accurate, among others, it is widely applied in theoretical research.

Chiorean [7] proposed a new formulation by which the biaxial interaction diagrams and moment capacity contours of a composite steel-concrete crosssection can be determined, which makes use of an incremental, iterative procedure based on arc-length constraint equations. These methods, based on the fiber approach, may lead to time-consuming in the structural analysis because an iterative solution is usually used in developing the yield and failure surface.

This paper presents an efficient method to generate yield and failure surfaces of the doubly symmetrical wide flange I-section under axial force combined with biaxial bending moments. The proposed method is implemented in a computer program, SPH that developed for nonlinear analysis of the 2D and 3D steel frames. The developed equations for yield and failure surfaces result in a reduction of the time-consuming process in comparison to the fiber method for the finite element analysis of the steel frame.

\section{Section geometry}

Consider the cross-section of I-shape with total height $h$, flange width $b_{f}$, flange thickness $t$, and web thickness $b_{w}$ subjected to the external bending moments about the $\mathrm{Y}$-axis and $\mathrm{Z}$-axis, and axial force in the $\mathrm{X}$-axis as shown in Fig. 1. Assumption can be made as follows: 1) the plane section remains plane after deformation; 2) shear and torsional interaction effects are not considered in the steel constitutive model.

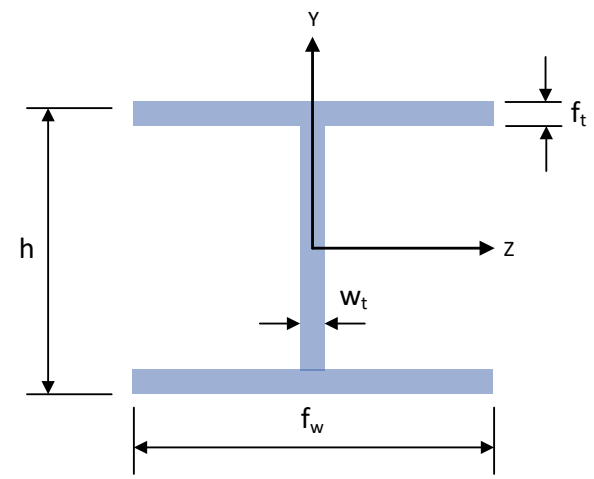

Fig. 1 I-section and dimensions

\section{Steel material}

The stress-strain relationship of steel material, both in tension and in compression, is assumed to be elastic-perfectly plastic, and the strain hardening of steel is neglected, as shown in Fig. 2. The normal stress in the section is calculated as:

$\sigma=E_{S} \varepsilon$

where $E_{s}$ is Young's modulus of steel; $\varepsilon$ is axial strain

The normal stress should not be greater than the yield strength of the steel material, $f_{y}$ :

$-f_{y} \leq \sigma \leq f_{y}$

The yield strength is determined as:

$f_{y}=E_{s} \varepsilon_{y}$

where $\varepsilon_{y}$ is yield axial strain. 


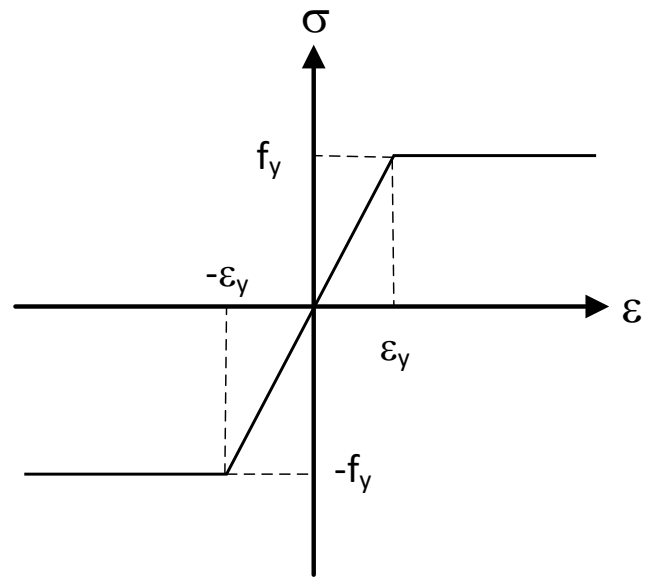

Fig. 2 The stress-strain relationship for steel

\section{Elastic limit surface}

The elastic limit surface separates the space inside the failure surface into two parts, elastic and plastic. This surface is established from a condition that normal stress at furthest location from the neutral axis of a cross-section reaches the yield strength as presented in the following equation:

$\sigma=\frac{P}{A}+\frac{M_{y}}{W_{y}}+\frac{M_{z}}{W_{z}}=f_{y}$

where $A$ is cross-section area; $W_{y}$ and $W_{z}$ are section moduli about $\mathrm{Y}$ and $\mathrm{Z}$ axes, respectively.

The following equation defines the elastic limit surface in terms of nondimensional quantities:

$p+m_{y e 0}+m_{z e 0}=1$

where:

$p=\frac{P}{P_{\max }} ; m_{y e 0}=\frac{M_{y e 0}}{M_{y e}} ; m_{z e 0}=\frac{M_{z e 0}}{M_{z e}}$

where $M_{y e}$ is maximum elastic moment about $\mathrm{Y}$-axis; $M_{z e}$ is maximum elastic moment about Z-axis; $M_{y e 0}$ is moment about $\mathrm{Y}$-axis on the elastic limit surface; $M_{z e 0}$ is moment about $\mathrm{Z}$-axis on the elastic limit surface; $P_{\max }$ is axial strength.

The maximum elastic moments are given by:

$M_{y e}=W_{y} f_{y} ; M_{z e}=W_{z} f_{y}$

The angle between the two components of bending moments is defined as:

$\tan \alpha=\frac{M_{y e 0}}{M_{z e 0}} \frac{I_{z}}{I_{y}}=\frac{m_{y e 0} M_{y e}}{m_{z e 0} M_{z e}} \frac{I_{z}}{I_{y}}$

where $I_{y}$ and $I_{z}$ are moments of inertia about $\mathrm{Y}$ and $\mathrm{Z}$ axes, respectively.

Rearranging Eq. (8) leads to:

$m_{y e 0}=\frac{m_{z e 0} M_{z e}}{M_{y e}} \frac{I_{y}}{I_{z}} \tan \alpha$

Substituting Eq. (9) into Eq. (5):

$m_{z e 0}=\frac{1-p}{M_{y e} I_{z}+M_{z e} I_{y} \tan \alpha} M_{y e} I_{z}$

Substituting Eq. (10) into Eq. (9):

$m_{y e 0}=\frac{1-p}{M_{y e} I_{z}+M_{z e} I_{y} \tan \alpha} M_{z e^{I}} I^{\tan \alpha}$
Finally, the moments on the elastic limit surface are calculated as:

$$
\begin{aligned}
& M_{y e 0}=\frac{1-p}{M_{y e} I_{z}+M_{z e} I_{y} \tan \alpha} M_{z e} I_{y} \tan \alpha M_{y e} \\
& M_{z e 0}=\frac{1-p}{M_{y e} I_{z}+M_{z e} I_{y} \tan \alpha} M_{y e} I_{z} M_{z e}
\end{aligned}
$$

Rearranging Eqs. (12) and (13) leads to simplified equations of the moments on the elastic limit surface:

$$
\begin{gathered}
M_{y e 0}=f_{y} \frac{1-p}{\frac{h}{b_{f}}+\tan \alpha} \tan \alpha W_{y} \\
M_{z e 0}=f_{y} \frac{1-p}{1+\frac{b_{f}}{h} \tan \alpha} W_{z}
\end{gathered}
$$

Since $P_{\max }, M_{y e}$, and $M_{z e}$ are constants for a cross-section, the limit elastic surface is represented by a plane as shown in Fig. 3.

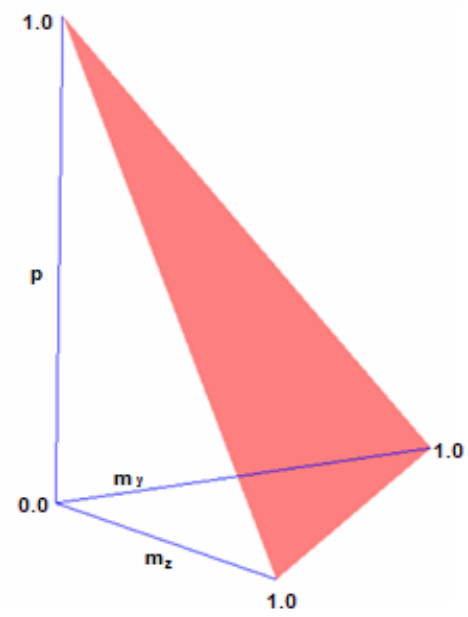

Fig. 3 The limit elastic surface

\section{Failure surface}

At failure, the centroid of the section lies on the neutral axis (NA) because the considered cross-section is doubly symmetrical, as shown in Fig. 4. Stresses over the cross-section reach to yield strength of steel (either compression or tension). The magnitude of the axial force determines the boundary between tension and compression regions. Notations of dimension are used to develop the equations of the failure surface are also shown in Fig. 4 for an I-section under biaxial loading. Based on the distribution of the stresses, the cross-section can be divided into 1) region of axial force which subjects to axial force and 2) regions of bending moment which subject to biaxial bending moment.

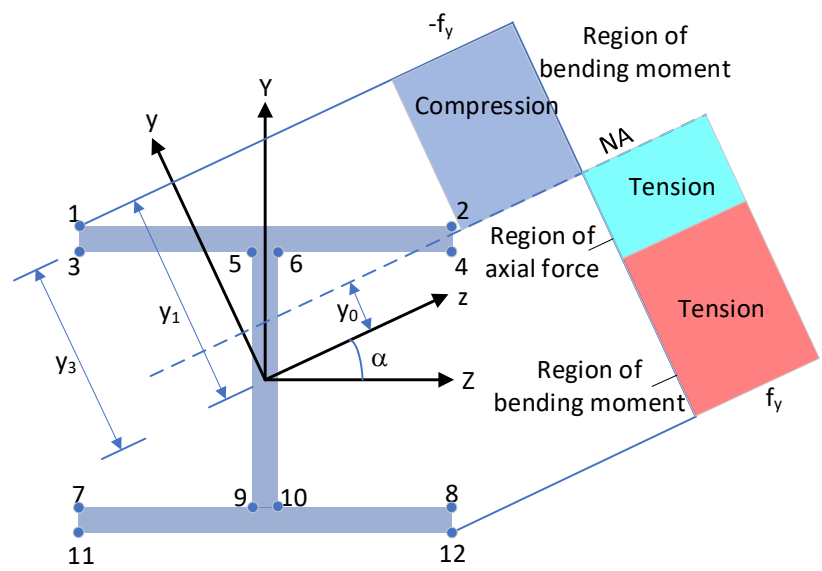

Fig. 4 I-section and dimensions 
A new coordinate system, $y z$ is defined by counterclockwise rotating the $Y Z$ coordinate system an angle of $\alpha$ where the y-axis is parallel to the resultant bending moment. The relationships between coordinates of two coordinate systems for a point on the cross-section are given by:

$z_{i}=Z_{i} \cos \alpha+Y_{i} \sin \alpha$

$y_{i}=-Z_{i} \sin \alpha+Y_{i} \cos \alpha$

where $Y_{i}$ and $Z_{i}$ are coordinates of the $\mathrm{i}^{\text {th }}$ point $(i=1 \div 12)$ in the $Y Z$ coordinate system; $y_{i}$ and $z_{i}$ are coordinates of the $\mathrm{i}^{\text {th }}$ point in the $y z$ coordinate system. The $y_{i}$ coordinate is also the distance from a point on the cross-section to the z-axis. Fig. 4 shows that the distance between the neutral axes before and after yielding is $y_{0}$

With a given axial force, $P$, and angle $\alpha$, the plastic bending moment on the failure surface can be computed by the following procedure:

- Determine axial force $P_{i}$, and the plastic bending moments $M_{y u, i}$, and $M_{z u, i}$ at the $\mathrm{i}^{\text {th }}$ point (Fig. 4) by using the equations shown in the appendix for different cases corresponding to an angle $\alpha$ and $y_{i}>0$ with $i=1 \div 12$, where the neutral axis is passing that point.

- Sort the bending moments and axial forces based on the axial forces from the smallest to the largest where $P_{i}<P_{i+1}, M_{y u, i}<M_{y u, i+1}$, $M_{z u, i}<M_{z u, i+1}$.

- Find $\mathrm{n}$ where $P_{n} \leq P<P_{n+1}$.

- Interpolate the distance from the center of the cross-section to the neutral axis, corresponding to the axial force, $P$, using the following equation:

$$
y=y_{n}+\frac{P-P_{n}}{P_{n+1}-P_{n}}\left(y_{n+1}-y_{n}\right)
$$

- Determine the plastic bending moment $M_{y u}, M_{z u}$ and axial force $\bar{P}$ from a distance $y$ calculated in Eq. (17) by using the equations in the appendix. If calculated axial force $\bar{P}$ is different from the axial force $P$, an iterative computation needs to conduct to find the distance $y$ which satisfied an error control $|(\bar{P}-P) / P| \leq 1 E-3$.

The failure surface is plotted in Fig. 5.

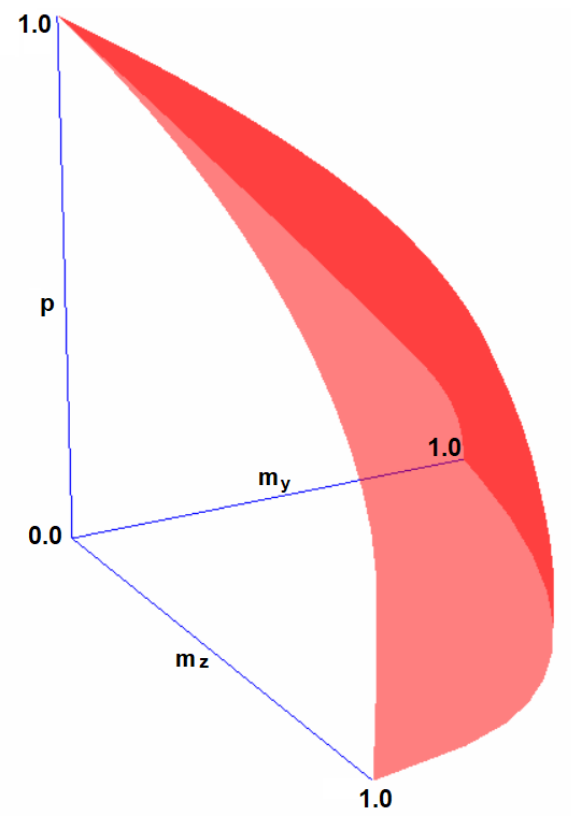

Fig. 5 Failure and yield surfaces

Examples of the failure surfaces are performed for the wide-flange Isections W12x30 (light-weight) and W14x426 (heavy-weight), respectively. The proposed method considered as an exact solution compared to the fiber method and Duan and Chen [1], as shown in Figs. 6 and 7. Fig. 6a shows the comparison of three solutions for the light-weight section W12x30 for the cases of $\mathrm{p}=0,0.5$, and 0.8 , and Fig. 7a is for the heavy-weight section $\mathrm{W} 14 \mathrm{x} 426$. Referring to those figures, the results of the proposed method are matched very well with the fiber method, while the results of Duan and Chen' method [1] are relatively agreed. The failure surfaces under the strong axis (Z-axis) obtained from the proposed method compared to the fiber and the Duan and Chen' method [1] in Figs. $6 \mathrm{~b}$ and $7 \mathrm{~b}$. All three methods produced very good agreement results except for the region of lower axial force where the Duan and Chen' method [1] is slightly less than those from the other methods. For the weak-axis (Y-axis) bending of wide-flange sections, it can be seen that the proposed method and two others' results are very close for both sections W12x30 and W14x426, as shown in Fig. 6d and 7d.

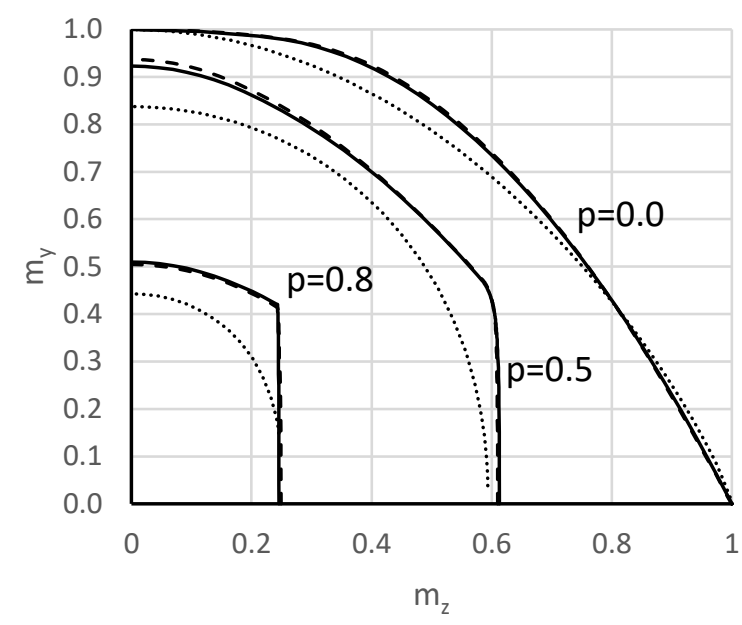

a) Plane view of the failure surfaces

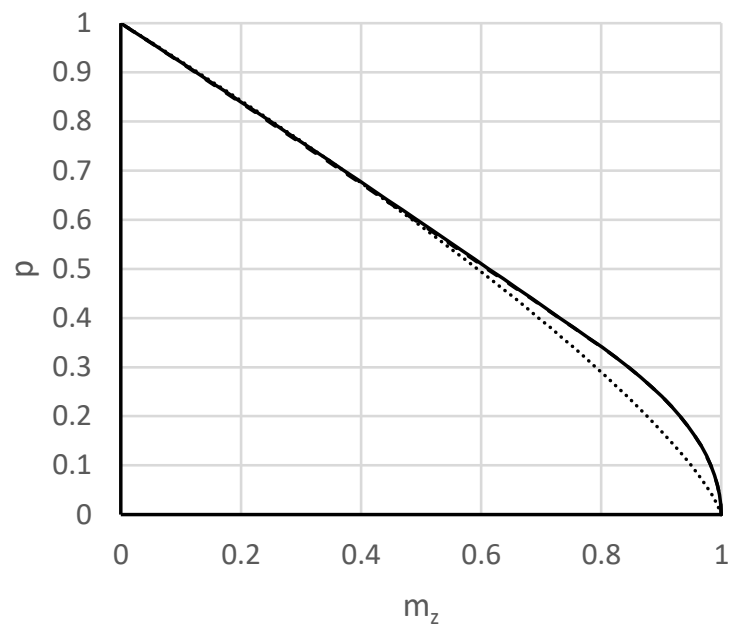

b) Failure surfaces under strong axis (Z-axis)

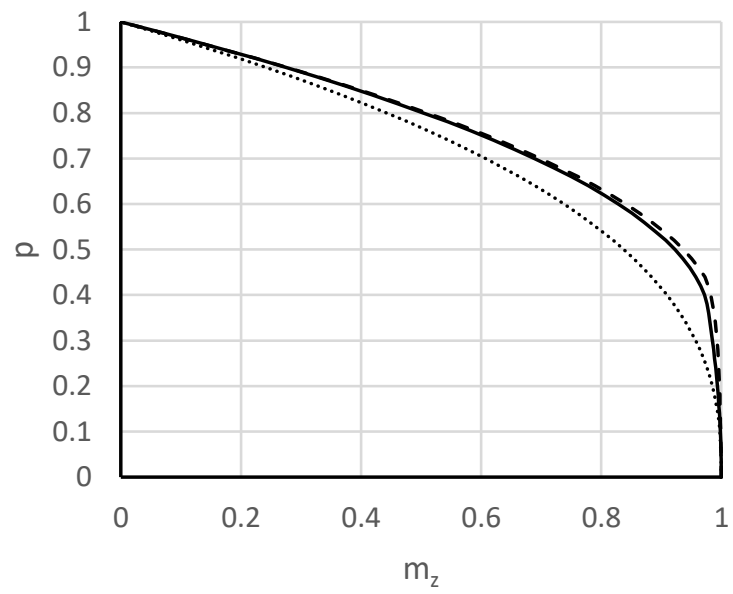

- - - - The fiber method

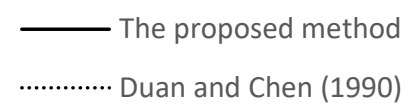

c) Failure surfaces under weak axis (Y-axis)

Fig. 6 Comparison of the failure surface for W12x30 


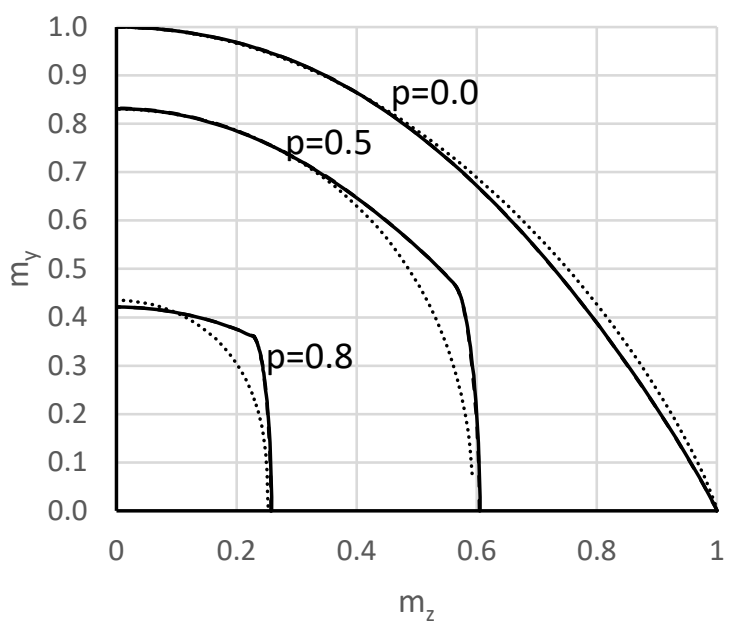

a) Plane view of the failure surfaces

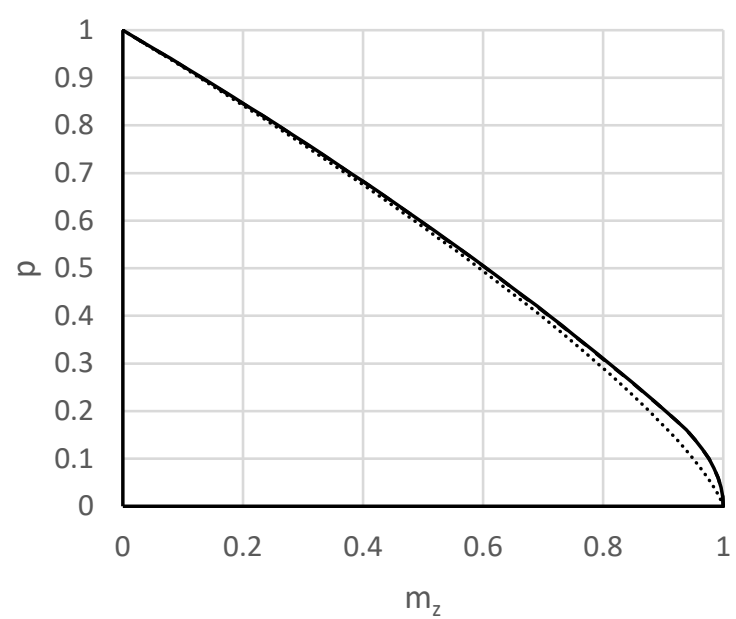

b) Failure surfaces under strong axis (Z-axis)

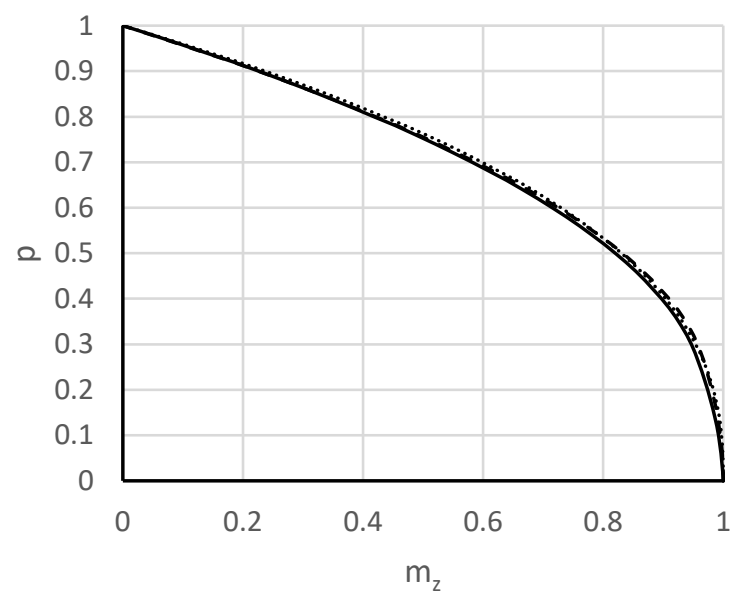

- - - - The fiber method

The proposed method

Duan and Chen (1990)

c) Failure surfaces under weak axis (Y-axis)

Fig. 7 Comparison of the failure surface for W14x426

\section{Yield surface}

The yield surfaces located within the elastic limit surface and the failure surface represent the gradual yielding of the cross-section. The relationship between bending moment and the unit rotation angle is nonlinear and can be approximated by hyperbolic curves, as shown in Fig. 8 and presented in the following equations:
If $M_{y u}>M_{y e 0}:$

$$
M_{y}=M_{y e 0}+\frac{\phi_{y}-\phi_{y e 0}}{\frac{1}{E I_{y}}+\frac{\phi_{y}-\phi_{y e 0}}{M_{y u}-M_{y e 0}}}
$$

If $M_{z u}>M_{z e 0}:$

$$
M_{z}=M_{z e 0}+\frac{\phi_{z}-\phi_{z e 0}}{\frac{1}{E I_{z}}+\frac{\phi_{z}-\phi_{z e 0}}{M_{z u}-M_{z e 0}}}
$$

If $M_{y u}<M_{y e 0}$ :

$$
M_{y}=M_{y e 0}-\frac{\phi_{y}-\phi_{y e 0}}{\frac{1}{E I_{y}}+\frac{\phi_{y}-\phi_{y e 0}}{M_{y e 0}-M_{y u}}}
$$

If $M_{z u}<M_{z e 0}:$

$$
M_{z}=M_{z e 0}-\frac{\phi_{z}-\phi_{z e 0}}{\frac{1}{E I_{z}}+\frac{\phi_{z}-\phi_{z e 0}}{M_{z e 0}-M_{z u}}}
$$

where $M_{y u}$ and $M_{z u}$ are plastic bending moments about y and $\mathrm{z}$ axes, respectively; $\phi_{y}$ and $\phi_{z}$ are unit rotation angles in $\mathrm{y}$ and $\mathrm{z}$ directions, respectively; and $\phi_{y e 0}$ and $\phi_{z e 0}$ are unit rotation angles at elastic limit of the section in $\mathrm{y}$ and $\mathrm{z}$ directions, respectively.

The curves for the moment-unit rotation angle relationship contain three segments: 1) straight line for the elastic state; 2) hyperbolic curve for yielding state; and 3) horizontal straight line for failure state.

The wide flange sections $\mathrm{W} 12 \times 30$ and $\mathrm{W} 14 \times 426$ are selected again to analyze and compare to the fiber method. The moment-unit rotation angle curves for the W12x30 section are plotted in Fig. 9 and Fig. 10. The curves from the proposed method match quite well with those from the fiber except the cases of $\mathrm{p}=0.5, \alpha=90^{0}$, and $\alpha=75^{\circ}$ where the proposed method produced slightly lower bending moment. Fig. 12 shows the moment-unit rotation angle curves for W14x426 section. Good agreements are also obtained from comparing those curves with the fiber method.

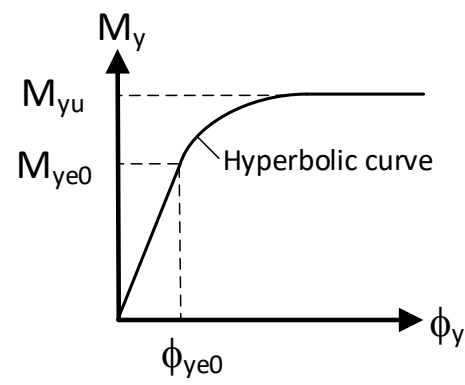

(a) $\mathrm{M}_{\mathrm{y}}-\phi_{\mathrm{y}}$;

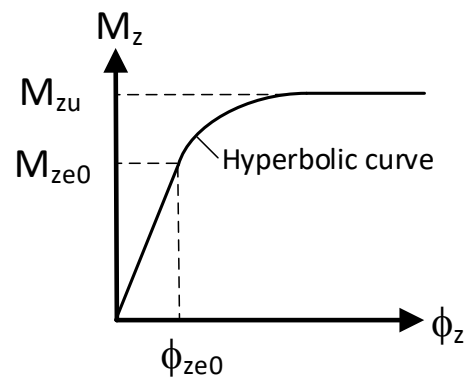

(b) $M_{z}-\phi_{z}$

Fig. 8 Moment-unit rotation angle relationship 


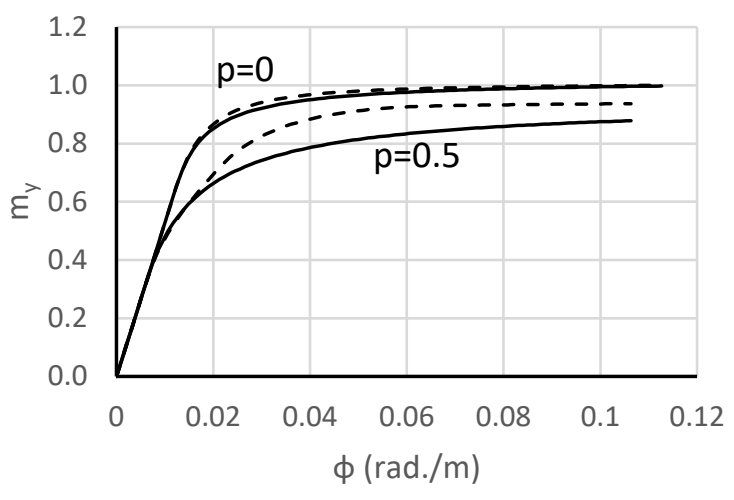

(a) $\alpha=90^{\circ}$

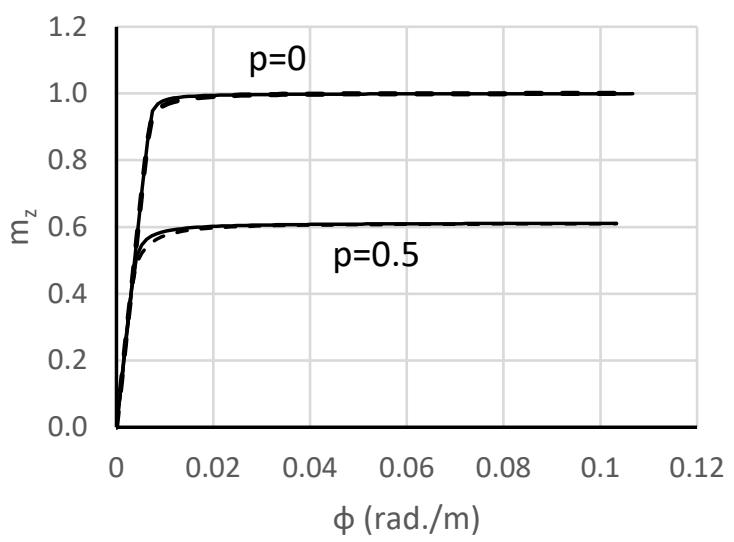

The proposed method - - - The fiber method

(b) $\alpha=0^{0}$

Fig. 9 Moment-unit rotation angle relationships for $\mathrm{W} 12 \times 30$ at $\alpha=90^{\circ}$ and $\alpha=0^{0}$
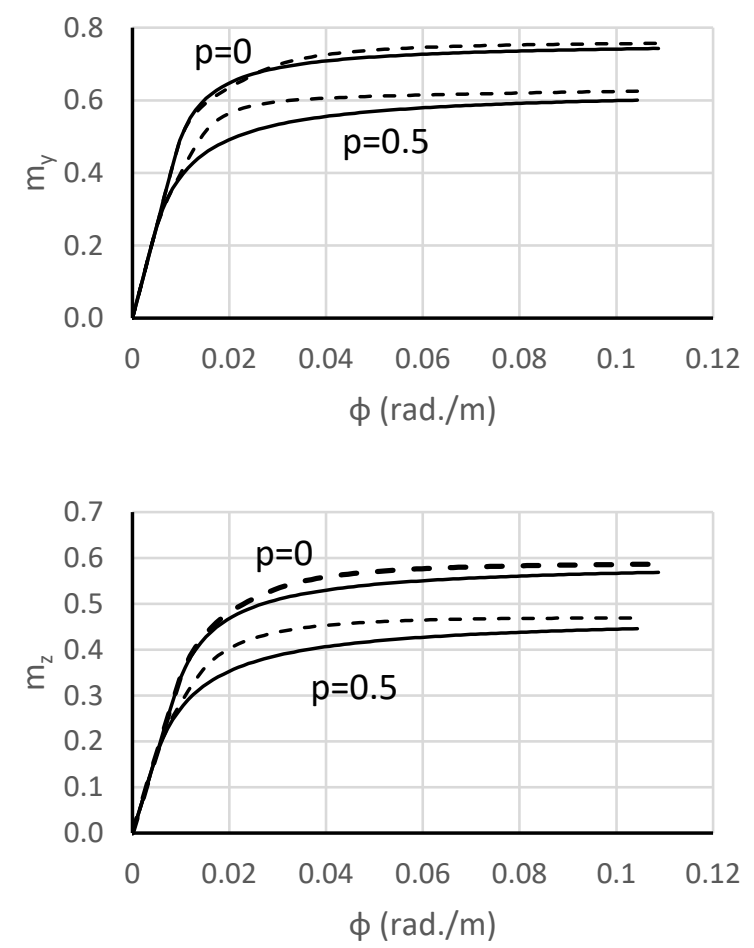

— The proposed method - - - The fiber method

Fig. 10 Moment-unit rotation angle relationships $\alpha=75^{\circ}$ for $\mathrm{W} 12 \times 30$

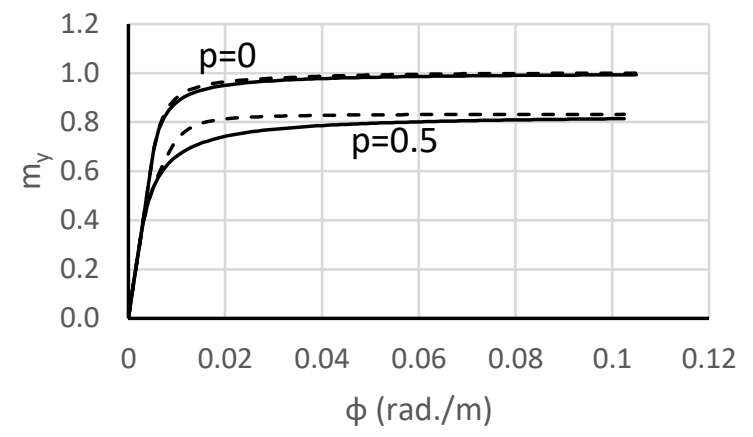

(a) $\alpha=90^{0}$

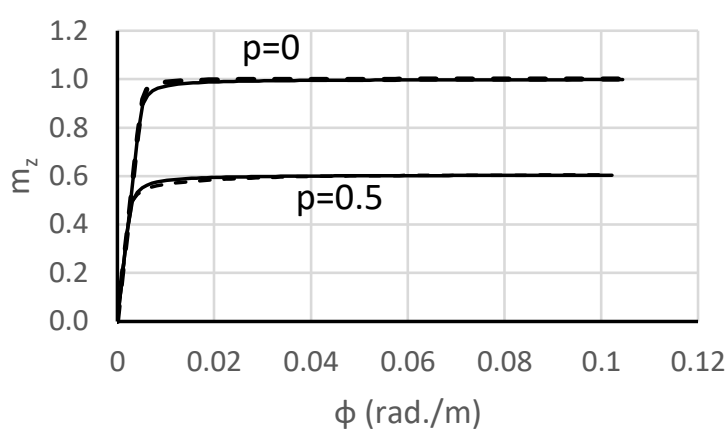

— The proposed method - - - The fiber method

(b) $\alpha=0^{0}$

Fig. 11 Moment-unit rotation angle relationships for W14x426 at $\alpha=90^{\circ}$ and $\alpha=0^{0}$
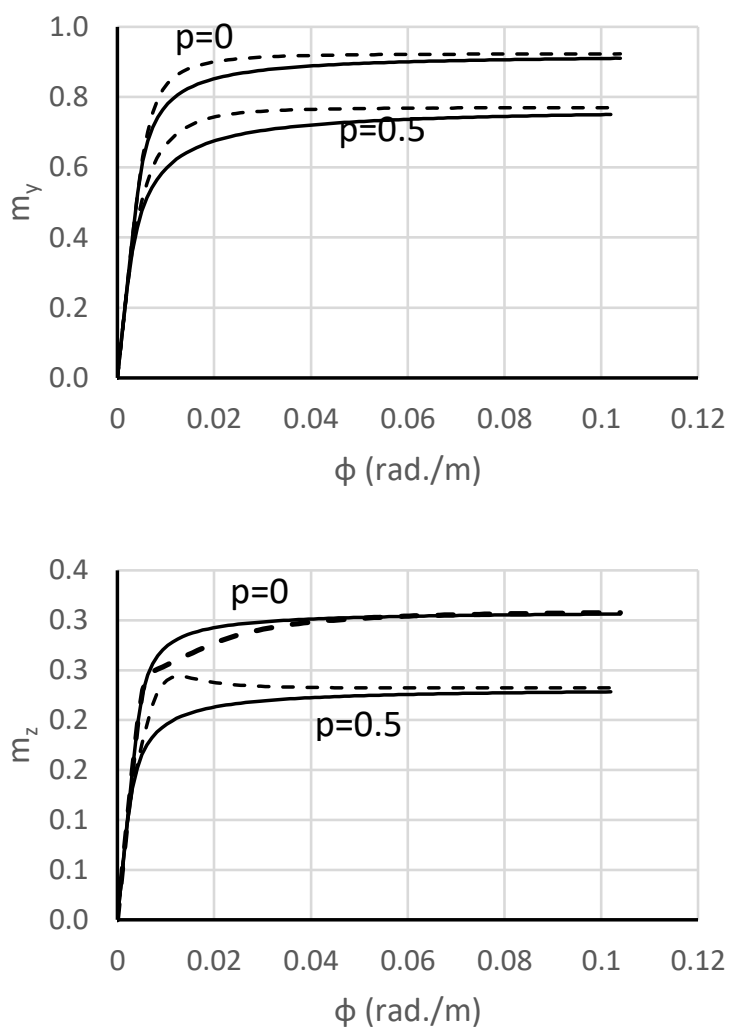

— The proposed method - - - The fiber method

Fig. 12 Moment-unit rotation angle relationships $\alpha=75^{\circ}$ for $\mathrm{W} 12 \times 30$

\section{Numerical analyses and results}

The following analyses for steel structures are conducted to verify the accuracy of the proposed method. The SPH program also can be used to predict the nonlinear behavior of plane and space frames. The proposed method is compared well to other solutions. 
A steel portal frame shown in Fig. 13 consists of a wide flange steel beam of W12x17 and two columns of W12x50. This frame has been analyzed by Liew et al. [8], Iu et al. [9], and Chiorean [10]. Plastic hinges are allowed to occur at only two ends of each element. In this study, the behavior of a steel frame is investigated. The geometry, section properties, and loading arrangement of the steel portal frame are depicted in Fig. 13. The frame is subjected to both vertical and lateral loading applied proportionally. The yield strength of all elements is 248.2 $\mathrm{MPa}$, and Young's modulus is E=20000 MPa. The nonlinear behaviors represented by the load-displacements curves at the point A computed by the proposed method and those given in Liew et al. [8], Iu et al. [9] and Chiorean [10] are compared in Fig. 14. It can be observed that the results agree very well.



Fig. 13 Steel portal frame

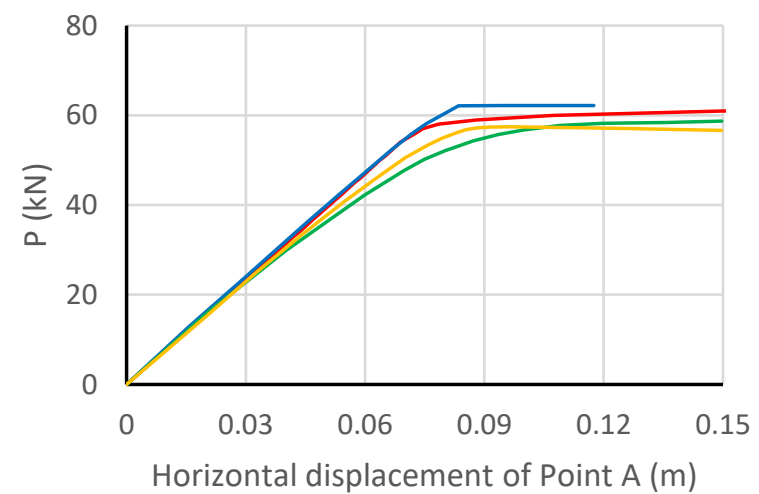

The proposed method

- Liew et al. (2001)

lu et al (2009)

Chiorean (2013)

Fig. 14 Load-displacement curve for point A of the steel portal frame

Fig. 15 shows Orbison's six-story space frame. This frame was analyzed by several researchers such as Liew et al. [11], Kim et al. [12,13], Kim and Choi [14], and Skordeli and Bisbos [15]. The yield strength of all members is 250 $\mathrm{MPa}$ (36 ksi) and Young's modulus is $206850 \mathrm{MPa}$ (30000 ksi). Uniform floor pressure of $4.8 \mathrm{KN} / \mathrm{m} 2$ ( $100 \mathrm{psf}$ ) is converted into equivalent concentrated loads on the top of the columns. Wind loads are simulated by point loads of $26.7 \mathrm{KN}$ (6 kips) in the $Y$-direction at every beam-column joints and only applied on the front elevation. The load-displacement curve at point $\mathrm{A}$ in the $\mathrm{Y}$-direction predicted by the proposed analysis compared well with that of Liew et al. [11], as shown in Fig. 16. The ultimate load factor calculated from the proposed method is 2.1 , that nearly equivalent to 2.062 calculated by Liew et al. [11].

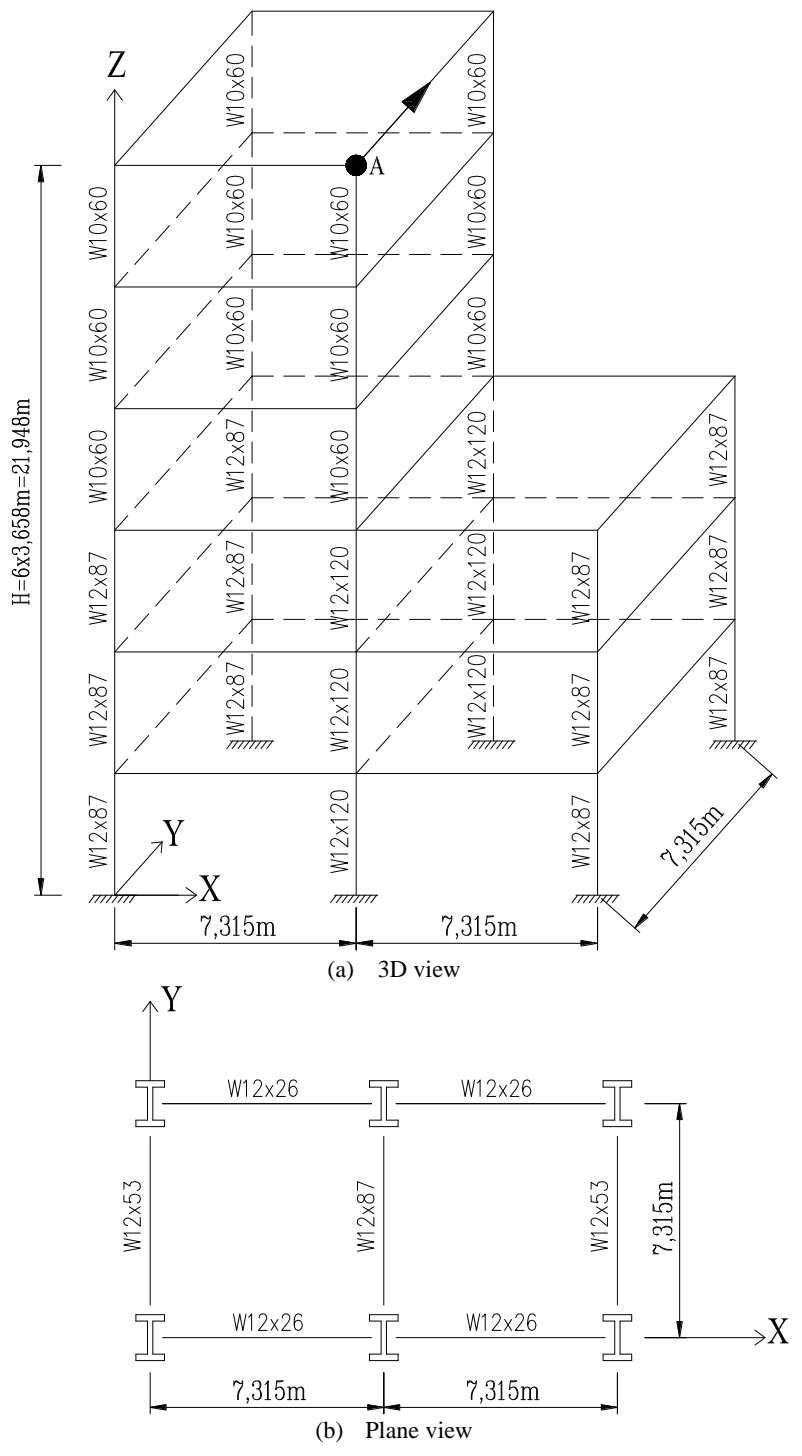

Fig. 15 Six-story space frame

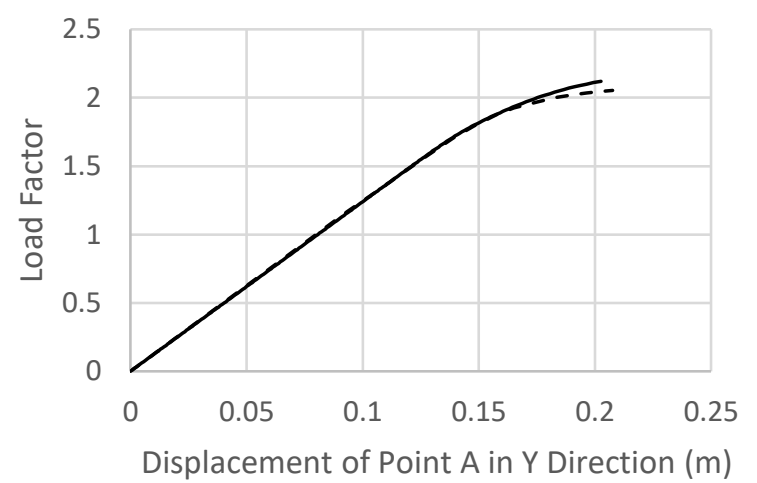

The proposed method - - - - Liew et al. (1990)

Fig. 16 The load-displacement curve at point $\mathrm{A}$ in the $\mathrm{Y}$ direction

\section{Conclusion}

A new method to develop the yield and failure surfaces for commonly used I-section in steel structure under biaxial loading is proposed. The equations of yield and failure surfaces are implemented in the nonlinear static analysis program SPH. Comparisons the predicted moment-unit rotation angle curves and failure surfaces with those from the fiber method confirm the validity of the proposed method. Load-displacement curves produced from the nonlinear analyses of the 2D and 3D steel frames using the proposed method agreed well with those from the available methods in the literature. The developed equations for yield and failure surfaces can help to save computational time in comparison to the fiber method. 


\section{Appendix}

- Case 1

If $y_{0} \geq y_{4} ; y_{0}<y_{2} ; y_{0}<y_{3}$ (Fig. A1)
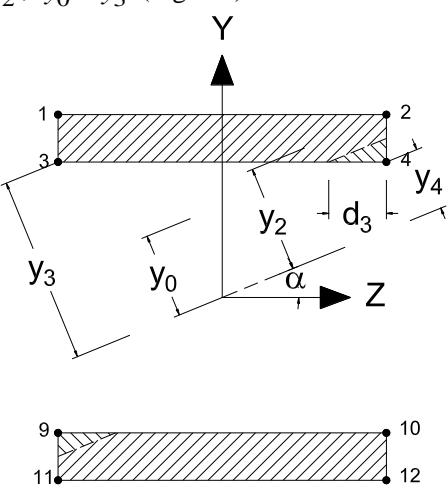

Fig. A1

$d_{3}=\left(y_{0}-y_{4}\right) / \sin \alpha$

$P=f_{y} d_{3}^{2} \tan \alpha$

$M_{Y}=f_{y} d_{3}^{2} \tan \alpha\left(\frac{b_{f}}{2}-\frac{d_{3}}{3}\right)$

$M_{Z}=f_{y}\left(b_{f} t(h-t)-d_{3}^{2} \tan \alpha\left(\frac{h}{2}-t+\frac{d_{3} \tan \alpha}{3}\right)\right)$

- $\quad$ Case 2

If $y_{2} \leq y_{0}<y_{3}$ (Fig. A2)
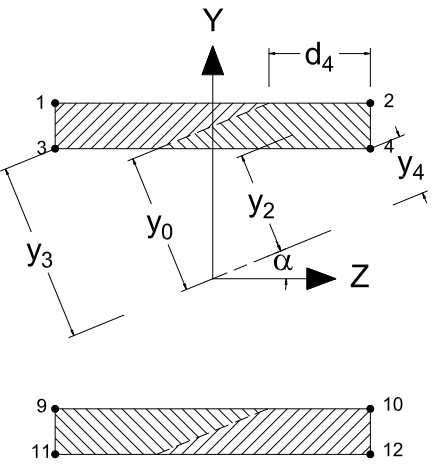

Fig. A2

$d_{4}=\left(y_{0}-y_{2}\right) / \sin \alpha$

$P=f_{y} t\left(\frac{t}{\tan \alpha}+2 d_{4}\right)$

$M_{Y}=f_{y} t\left(\left(b_{f}-d_{4}-\frac{t}{\tan \alpha}\right)\left(d_{4}+\frac{t}{\tan \alpha}\right)\right)$

$+f_{y} \frac{t^{2}}{\tan \alpha}\left(d_{4}+\frac{2 t}{3 \tan \alpha}-\frac{b_{f}}{2}\right)$

$M_{Z}=f_{y}\left(t\left(b_{f}-d_{4}-\frac{t}{\tan \alpha}\right)(h-t)+\frac{t^{2}}{\tan \alpha}\left(\frac{h}{2}-\frac{t}{3}\right)\right)$

\section{- $\quad$ Case 3}

If $y_{3} \leq y_{0}<y_{1}$ (Fig. A3)
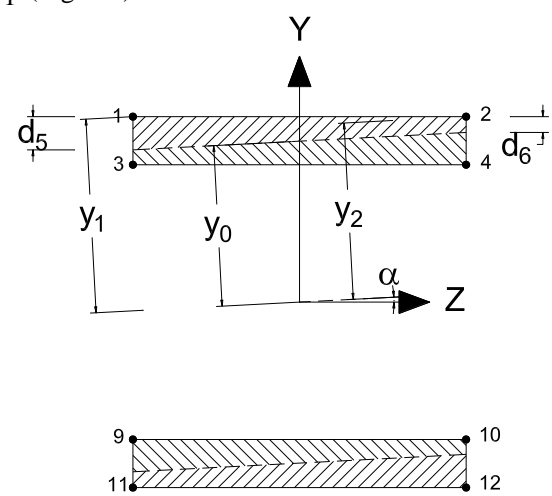

Fig. A3 $d_{5}=\left(y_{1}-y_{0}\right) / \cos \alpha ; d_{6}=\left(y_{2}-y_{0}\right) / \cos \alpha$

$P=2 f_{y} b_{f}\left(t-\frac{d_{5}+d_{6}}{2}\right)$

$M_{Y}=f_{y}\left(d_{5}-d_{6}\right) \frac{b_{f}^{2}}{6}$

$M_{Z}=f_{y}\left(d_{6} b_{f}\left(h-d_{6}\right)+\left(d_{5}-d_{6}\right) b_{f}\left(\frac{h}{2}-d_{6}-\frac{d_{5}-d_{6}}{3}\right)\right)$

- $\quad$ Case 4

If $y_{3} \leq y_{0}<y_{2}$ (Fig. A4)

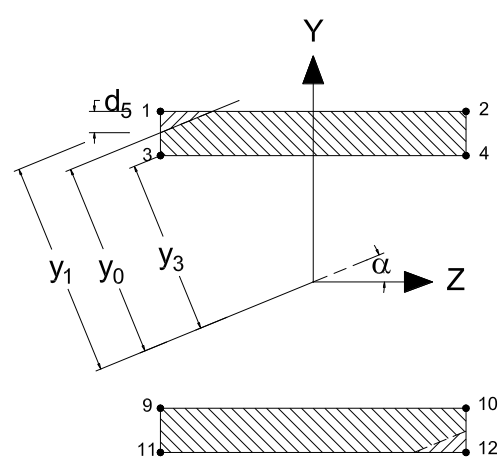

Fig. A4

$d_{5}=\left(y_{1}-y_{0}\right) / \cos \alpha$

$P=2 f_{y}\left(b_{f} t-\frac{1}{2} \frac{d_{5}^{2}}{\tan \alpha}\right)$

$M_{Y}=f_{y} \frac{d_{5}^{2}}{\tan \alpha}\left(\frac{b_{f}}{2}-\frac{d_{5}}{3 \tan \alpha}\right)$

$M_{Z}=f_{y} \frac{d_{5}^{2}}{\tan \alpha}\left(\frac{h}{2}-\frac{d_{5}}{3}\right)$

- $\quad$ Case 5

If $y_{2} \leq y_{0}<y_{3}$ and $\frac{h-2 t}{b_{f}} \leq \tan \alpha<\frac{h}{b_{f}}$ (Fig. A5)
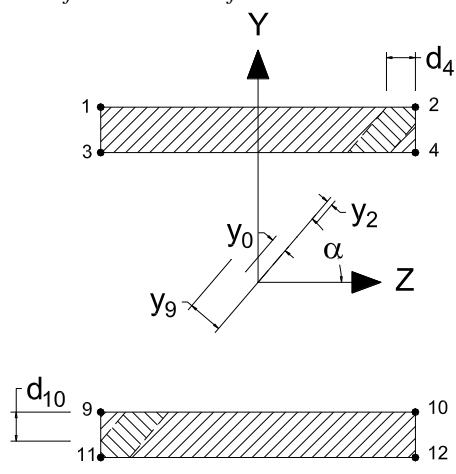

Fig. A5

$d_{4}=\left(y_{0}-y_{2}\right) / \sin \alpha ; d_{10}=\left(y_{9}-y_{0}\right) / \cos \alpha$

$$
\begin{aligned}
P= & f_{y} t\left(\frac{t}{\tan \alpha}+2 d_{4}\right)-f_{y} \frac{d_{10}^{2}}{\tan \alpha} \\
M_{Y}= & 2 f_{y} t\left(b_{f}-d_{4}-\frac{t}{\tan \alpha}\right)\left(\frac{d_{4}}{2}+\frac{t}{2 \tan \alpha}\right) \\
& +f_{y} \frac{t^{2}}{\tan \alpha}\left(d_{4}+\frac{2 t}{3 \tan \alpha}-\frac{b_{f}}{2}\right) \\
& +f_{y} \frac{d_{10}^{2}}{\tan \alpha}\left(\frac{b_{f}}{2}-\frac{d_{10}}{3 \tan \alpha}\right) \\
M_{Z}= & f_{y}\left(t\left(b_{f}-d_{4}-\frac{t}{\tan \alpha}\right)(h-t)+\frac{t^{2}}{\tan \alpha}\left(\frac{h}{2}-\frac{t}{3}\right)\right) \\
& -f_{y} \frac{d_{10}^{2}}{\tan \alpha}\left(\frac{h}{2}-t+\frac{d_{10}}{3}\right)
\end{aligned}
$$




\section{- Case 6}

If $y_{2} \leq y_{0}<y_{3}$ and $\frac{h}{b_{f}} \leq \tan \alpha$ (Fig. A6)

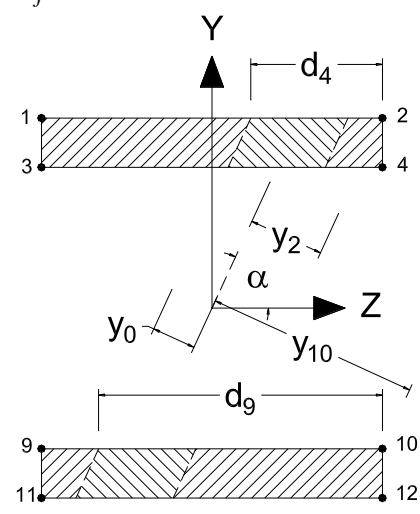

Fig. A6

$d_{4}=\left(y_{0}-y_{2}\right) / \sin \alpha ; d_{9}=\left(y_{0}-y_{10}\right) / \sin \alpha$

$$
\begin{aligned}
P= & f_{y} t\left(\frac{t}{\tan \alpha}+2 d_{4}\right)-f_{y} t\left(2 b_{f}-\frac{t}{\tan \alpha}-2 d_{9}\right) \\
M_{Y} & =f_{y} t\left(\left(b_{f}-d_{4}-\frac{t}{\tan \alpha}\right)\left(d_{4}+\frac{t}{\tan \alpha}\right)\right) \\
& +f_{y} \frac{t^{2}}{\tan \alpha}\left(d_{4}+\frac{2 t}{3 \tan \alpha}-\frac{b_{f}}{2}\right) \\
& +f_{y} t\left(\left(b_{f}-d_{9}-\frac{t}{\tan \alpha}\right)\left(d_{9}+\frac{t}{\tan \alpha}\right)\right) \\
& +f_{y} \frac{t^{2}}{\tan \alpha}\left(d_{9}+\frac{2 t}{3 \tan \alpha}-\frac{b_{f}}{2}\right) \\
M_{Z} & =f_{y} t(h-t)\left(d_{9}-d_{4}\right)
\end{aligned}
$$

\section{- $\quad$ Case 7}

If $y_{0} \leq y_{6}$ and $y_{0} \geq y_{7}$ (Fig. A7)

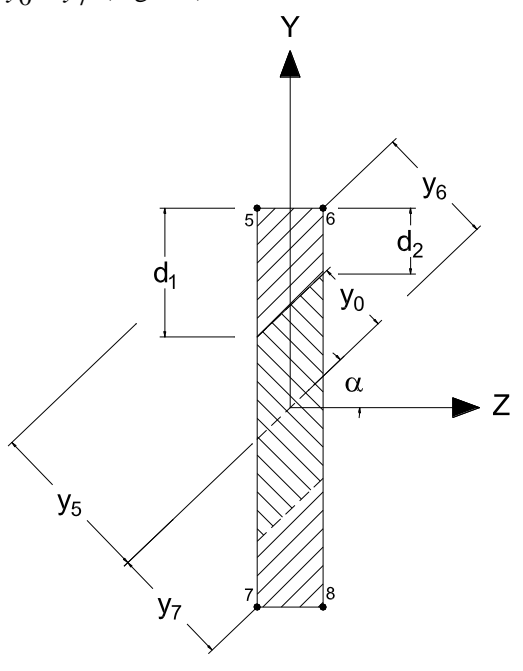

Fig. A7

$d_{1}=\left(y_{5}-y_{0}\right) / \cos \alpha ; d_{2}=\left(y_{6}-y_{0}\right) / \cos \alpha$

$P=f_{y} b_{w}\left(h-2 t-\left(d_{1}+d_{2}\right)\right)$

$M_{Y}=\frac{1}{6} f_{y} b_{w}^{3} \tan \alpha$

$M_{Z}=f_{y} d_{2} b_{w}\left(h-2 t-d_{2}\right)$

$$
+f_{y} b_{w}^{2} \tan \alpha\left(\frac{h}{2}-t-d_{2}-\frac{b_{w} \tan \alpha}{3}\right)
$$

\section{- $\quad$ Case 8}

If $y_{0} \leq y_{5}, y_{0}>y_{6}$, and $y_{0}<y_{7}$ (Fig. A8)

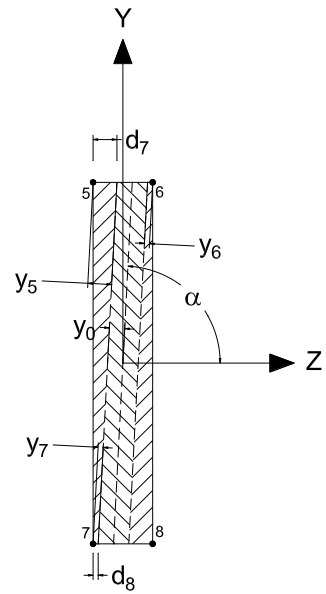

Fig. $A 8$

$d_{7}=\left(y_{5}-y_{0}\right) / \sin \alpha ; d_{8}=\left(y_{7}-y_{0}\right) / \sin \alpha$

$P=f_{y}\left(b_{w}-d_{7}-d_{8}\right)(h-2 t)$

$M_{Y}=f_{y} d_{8}\left(b_{w}-d_{8}\right)(h-2 t)$

$+f_{y}\left(d_{7}-d_{8}\right)(h-2 t)\left(\frac{b_{w}}{2}-\frac{d_{7}+2 d_{8}}{3}\right)$

$M_{Z}=\frac{1}{6} f_{y}\left(\left(d_{7}-d_{8}\right)(h-2 t)^{2}\right)$

- $\quad$ Case 9

If $y_{0} \leq y_{5}, y_{0}>y_{6}$, and $y_{0}>y_{7}$ (Fig. A9)

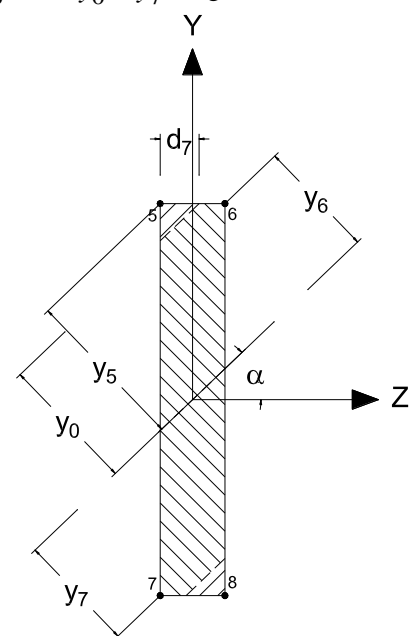

Fig. A9

$$
\begin{aligned}
& d_{7}=\left(y_{5}-y_{0}\right) / \sin \alpha \\
& P=f_{y}\left(b_{w}(h-2 t)-d_{7}^{2} \tan \alpha\right) \\
& M_{Y}=f_{y} d_{7}^{2} \tan \alpha\left(\frac{b_{w}}{2}-\frac{d_{7}}{3}\right) \\
& M_{Z}=f_{y} d_{7}^{2} \tan \alpha\left(\frac{h}{2}-t-\frac{d_{7} \tan \alpha}{3}\right)
\end{aligned}
$$

\section{References}

[1] Duan, L., and Chen, W.F., (1990), "A yield surface equation for doubly symmetrical sections", Eng. Struc., 12; 114-119. https://doi.org/10.1016/0141-0296(90)90016-L

[2] Orbison, J. G., McGuire, M., and Abel, J.F. (1982), "Yield surface application in nonlinear steel frame analysis", Computer methods in applied mechanics and engineering, 33; 557-573. https://doi.org/10.1016/0045-7825(82)90122-0

[3] Taucer, F., Spacone, E., and Filippou, F.C. (1991). A Fiber Beam-Column Element for Seismic Response Analysis of Reinforced Concrete Structures. Berkekey, California: Earthquake Engineering Research Center, College of Engineering, University of California, 91(17)

[4] Ngo Huu, C. (2006), "Practical advanced analysis of steel-concrete composite structures using fiber-hinge method", Ph.D. dissertation, Department of Civil and Environmental Engineering, the Graduate school of Sejong University.

[5] Ngo, H.C., and Kim, S.E. (2009), "Practical advanced analysis of space steel frames using fiber hinge method", Thin-Walled Structures, 47(4), 421-430.

https://doi.org/10.1016/j.tws.2008.08.007 
[6] Nguyen, P.C., and Kim, S.E., (2015), "Second-order spread-of-plasticity approach for nonlinear time-history analysis of space semi-rigid steel frames", Finite Elements in Analysis and Design, 105:1-15. https://doi.org/10.1016/j.finel.2015.06.006

[7] Chiorean, C.G. (2010), "Computerised interaction diagrams and moment capacity contours for composite steel-concrete cross-sections", Eng. Struc., 32; 3734-3757. https://doi.org/10.1016/i.engstruct.2010.08.019

[8] Liew, J. R., Chen, H., and Shanmugam, N. E. (2001), "Inelastic analysis of steel frames with composite beams", Journal of Structural Engineering, 127(2), 194-202. https://doi.org/10.1016/S0141-0296(99)00085-1

[9] Iu, C. K., Bradford, M.A., \& Chen, W.F. (2009), "Second-order inelastic analysis of composite framed structures based on the refined plastic hinge method", Eng. Struc., 31(3), 799-813. https://doi.org/10.1016/j.engstruct.2008.12.007

[10] Chiorean, C.G. (2013), "A computer method for nonlinear inelastic analysis of 3D composite $\begin{array}{llll}\text { steel-concrete frame } & \text { Structures", Eng. } & \text { Struc., 57, } & \end{array}$ https://doi.org/10.1016/i.engstruct. 2013.09.025

[11] Liew, J.R., Chen, H., Shanmugam, N.E. and Chen, W.F. (2000), "Improved nonlinear plastic hinge analysis of space frame structures", Eng. Struc., 22(10), pp.1324-1338. https://doi.org/10.1016/S0141-0296(99)00085-1

[12] Kim, S.E., Kim, Y. and Choi, S.H. (2001a), "Nonlinear analysis of 3-D steel frames", Thinwalled structures, 39(6), pp.445-461. https://doi.org/10.1016/S0263-8231(01)00017-9

[13] Kim, S.E., Park, M.H. and Choi, S.H., (2001b), "Direct design of three-dimensional frames using practical advanced analysis", Eng. Struc., 23(11), pp.1491-1502. https://doi.org/10.1016/S0141-0296(01)00041-4

[14] Kim, S.E. and Choi, S.H., (2001), "Practical advanced analysis for semi-rigid space frames", International journal of solids and structures, 38(50-51), pp.9111-9131. https://doi.org/10.1016/S0020-7683(01)00141-X

[15] Skordeli, M.A. and Bisbos, C.D., (2010), "Limit and shakedown analysis of 3D steel frames via approximate ellipsoidal yield surfaces", Eng. Struc., 32(6), pp.1556-1567. https://doi.org/10.1016/j.engstruct.2010.02.004 